\title{
A scheme for the teleportation of multiqubit quantum information via the control of many agents in a network
}

\author{
Chui-Ping Yang and Siyuan Han \\ Department of Physics and Astronomy, University of Kansas, Lawrence,
} Kansas 66045, USA

\begin{abstract}
We present a new scheme for teleporting multiqubit quantum information from a sender to a distant receiver via the control of many agents in a network. We show that the receiver can successfully restore the original state of each qubit as long as all the agents cooperate. However, it is remarkable that for certain type of teleported states, the receiver can not gain any amplitude information even if one agent does not collaborate. In addition, our analysis shows that for general input states of each message qubit, the average fidelity for the output states, when even one agent does not take action, is the same as that for the previous proposals.
\end{abstract}

PACS numbers: 03.67.Lx, 03.65.-w

In 1993 Bennett et al. showed that an unknown quantum state of a two-state particle can be teleported from one place to another [1]. Since then, much progress has been made in this area. Experimentally, quantum teleportation was recently demonstrated using photon polarization states [2], optical coherent states [3], and nuclear magnetic resonance [4]. On the other hand, Karlsson and Bourennane [5] and Hillery et. al. [6] generalized the idea of Bennett et al. to the "one to many" quantum communication of teleportation via many-particle quantum channels, i.e., quantum secret sharing - splitting a message among many spatially-separated agents in a network so that no subset of agents is sufficient to read the message but the entire set is. Thereafter, a lot of works on quantum secret sharing were presented [7-12].

Recently, there is an increasing interest in the controlled teleportation. Different from the secret sharing, the controlled teleportation is actually the "one to one (a sender to a distant receiver)" quantum information transfer via the control of agents in a network. The controlled teleportation is useful in the context of quantum information such as networked quantum information processing and cryptographic conferencing [13,14]. And also, it may have other interesting applications, such as in opening a credit account on the agreement of all the managers in a network. Recently, several schemes have been proposed $[15,16]$ for controlled teleportation. As relevant to this work, we may cite the works in Refs. [5,6], and the recent work in Ref. [15] on simultaneous teleportation of multiqubit quantum information via the control of many agents in a network. The distinct feature of the scheme described in [15] is that compared with the method in $[5,6]$, the resources required for auxiliary qubits, local operations, and classical communication are greatly reduced. However, as addressed in [15], when the teleported message qubit $i$ is initially in an arbitrary state $\alpha_{i}|0\rangle+\beta_{i}|1\rangle$, the resulting density operator for the corresponding qubit $i^{\prime \prime}$ belonging to the receiver would be

$$
\rho_{i^{\prime \prime}}=\left|\alpha_{i}\right|^{2}|0\rangle\left\langle\left. 0|+| \beta_{i}\right|^{2} \mid 1\right\rangle\langle 1|
$$

or

$$
\rho_{i^{\prime \prime}}=\left|\alpha_{i}\right|^{2}|1\rangle\left\langle\left. 1|+| \beta_{i}\right|^{2} \mid 0\right\rangle\langle 0|
$$

(depending on the sender's Bell-state measurement outcome), when one agent does not collaborate. This result shows that the receiver can obtain amplitude information about the sender's each message qubit even though he/she knows nothing about its phase. We note that the methods in $[5,6]$ also have this shortcoming since the same results (1) and (2) can be obtained if not all agents collaborate (for the details, see reference [15]). Therefore, it is not secure to employ the previous methods to realize the controlled teleportation provided information is encoded through amplitude.

In this letter we will present a scheme for realizing the simultaneous teleportation of multiqubit quantum information from a sender to a distant receiver via the control of many agents in a network. We will show that using this scheme, the receiver can restore the original state of each message qubit when all the agents cooperate. However, it is interesting to note that for certain kinds of teleported states, the receiver cannot gain any amplitude information even if one agent does not collaborate. In addition, our analysis shows that for general input states of each message qubit, the average fidelity of the output states, when even one agent does not cooperate, is the same as that of the previous proposals $[5,6,15]$.

Consider that Alice has a string of message qubits labeled by $1,2, \ldots, m$, which is initially in the state $\prod_{i=1}^{m}\left(\alpha_{i}|0\rangle_{i}+\right.$ $\left.\beta_{i}|1\rangle_{i}\right)$. To simplify our presentation, we denote $\left|\varphi_{i}\right\rangle \equiv \alpha_{i}|0\rangle_{i}+\beta_{i}|1\rangle_{i}$ in the following. Suppose that Alice wishes to send the $m$-qubit information to Bob via the control of $n$ agents $\left(A_{1}, A_{2}, \ldots, A_{n}\right)$ in a network, such that Bob can get 
the complete information of each message qubit only if all the agents collaborate. This task can be implemented with the following prescription:

Firstly, Alice prepares the following EPR-GHZ entangled state through local logic gates

$$
\prod_{i=1}^{m}|\mathrm{EPR}\rangle_{i^{\prime} i^{\prime \prime}} \otimes|\mathrm{GHZ}\rangle_{+}+\prod_{i=1}^{m}|\widetilde{\mathrm{EPR}}\rangle_{i^{\prime} i^{\prime \prime}} \otimes|\mathrm{GHZ}\rangle_{-},
$$

where $|\mathrm{EPR}\rangle_{i^{\prime} i^{\prime \prime}}=|00\rangle_{i^{\prime} i^{\prime \prime}}+|11\rangle_{i^{\prime} i^{\prime \prime}}$ and $|\widetilde{\mathrm{EPR}}\rangle_{i^{\prime} i^{\prime \prime}}=|01\rangle_{i^{\prime} i^{\prime \prime}}-|10\rangle_{i^{\prime} i^{\prime \prime}}$ are EPR states for the qubit pair $\left(i^{\prime}, i^{\prime \prime}\right)$, and $|\mathrm{GHZ}\rangle_{+}=|0\rangle^{\otimes n}|0\rangle_{a} \pm|1\rangle^{\otimes n}|1\rangle_{a}$ are $(n+1)$-qubit GHZ states. Then, Alice sends the first $n$ GHZ qubits to the $n$ agents and the $m$ EPR qubits $\left(1^{\prime \prime}, 2^{\prime \prime}, \ldots, m^{\prime \prime}\right)$ to Bob, while keeping the last GHZ qubit (labeled by $a$ ) and the other $m$ EPR qubits $\left(1^{\prime}, 2^{\prime}, \ldots, m^{\prime}\right)$ to herself. The state of the whole system is given by

$$
\prod_{i=1}^{m}\left|\varphi_{i}\right\rangle|\mathrm{EPR}\rangle_{i^{\prime} i^{\prime \prime}} \otimes|\mathrm{GHZ}\rangle_{+}+\prod_{i=1}^{m}\left|\varphi_{i}\right\rangle|\widetilde{\mathrm{EPR}}\rangle_{i^{\prime} i^{\prime \prime}} \otimes|\mathrm{GHZ}\rangle_{-},
$$

which can be expressed as

$$
\begin{aligned}
& \prod_{i=1}^{m}\left[\left|\phi_{i i^{\prime}}^{+}\right\rangle\left(\alpha_{i}|0\rangle_{i^{\prime \prime}}+\beta_{i}|1\rangle_{i^{\prime \prime}}\right)+\left|\phi_{i i^{\prime}}^{-}\right\rangle\left(\alpha_{i}|0\rangle_{i^{\prime \prime}}-\beta_{i}|1\rangle_{i^{\prime \prime}}\right)\right. \\
& \left.+\left|\psi_{i i^{\prime}}^{+}\right\rangle\left(\alpha_{i}|1\rangle_{i^{\prime \prime}}+\beta_{i}|0\rangle_{i^{\prime \prime}}\right)+\left|\psi_{i i^{\prime}}^{-}\right\rangle\left(\alpha_{i}|1\rangle_{i^{\prime \prime}}-\beta_{i}|0\rangle_{i^{\prime \prime}}\right)\right] \\
& \otimes|\mathrm{GHZ}\rangle_{+} \\
& +\prod_{i=1}^{m}\left[\left|\phi_{i i^{\prime}}^{+}\right\rangle\left(\alpha_{i}|1\rangle_{i^{\prime \prime}}-\beta_{i}|0\rangle_{i^{\prime \prime}}\right)+\left|\phi_{i i^{\prime}}^{-}\right\rangle\left(\alpha_{i}|1\rangle_{i^{\prime \prime}}+\beta_{i}|0\rangle_{i^{\prime \prime}}\right)\right. \\
& \left.+\left|\psi_{i i^{\prime}}^{+}\right\rangle\left(-\alpha_{i}|0\rangle_{i^{\prime \prime}}+\beta_{i}|1\rangle_{i^{\prime \prime}}\right)+\left|\psi_{i i^{\prime}}^{-}\right\rangle\left(-\alpha_{i}|0\rangle_{i^{\prime \prime}}-\beta_{i}|1\rangle_{i^{\prime \prime}}\right)\right] \\
& \otimes|\mathrm{GHZ}\rangle_{-} .
\end{aligned}
$$

Above and below, the subscripts $i i^{\prime}=11^{\prime}, 22^{\prime}, 33^{\prime} \ldots ; i^{\prime} i^{\prime \prime}=1^{\prime} 1^{\prime \prime}, 2^{\prime} 2^{\prime \prime}, 3^{\prime} 3^{\prime \prime} \ldots ;$ and $i^{\prime \prime}=1^{\prime \prime}, 2^{\prime \prime}, 3^{\prime \prime} \ldots ;$ for $i=1,2,3 \ldots$ In addition, normalization factors throughout this letter are omitted for simplicity. The states $\left|\phi_{i i^{\prime}}^{+}\right\rangle,\left|\phi_{i i^{\prime}}^{-}\right\rangle,\left|\psi_{i i^{\prime}}^{+}\right\rangle$, and $\left|\psi_{i i^{\prime}}^{-}\right\rangle$related to Eq. (5) are the four Bell states for the qubit pair $\left(i, i^{\prime}\right)$, taking the form of

$$
\begin{aligned}
\left|\phi_{i i^{\prime}}^{ \pm}\right\rangle & =|00\rangle_{i i^{\prime}} \pm|11\rangle_{i i^{\prime}}, \\
\left|\psi_{i i^{\prime}}^{ \pm}\right\rangle & =|01\rangle_{i i^{\prime}} \pm|10\rangle_{i i^{\prime}} .
\end{aligned}
$$

Secondly, Alice performs a series of two-qubit Bell-state measurements respectively on $m$ qubit pairs $\left(1,1^{\prime}\right)$, $\left(2,2^{\prime}\right), \ldots\left(m, m^{\prime}\right)$. After that, one has

$$
|\psi\rangle|\mathrm{GHZ}\rangle_{+}+\left|\psi^{\prime}\right\rangle|\mathrm{GHZ}\rangle_{-},
$$

where $|\psi\rangle$ and $\left|\psi^{\prime}\right\rangle$ are the states for the $m$ qubits $\left(1^{\prime \prime}, 2^{\prime \prime}, \ldots, m^{\prime \prime}\right)$ belonging to Bob, which are expressed as follows

$$
|\psi\rangle=\prod_{i=1}^{m}|\psi\rangle_{i^{\prime \prime}}, \quad\left|\psi^{\prime}\right\rangle=\prod_{i=1}^{m}\left|\psi^{\prime}\right\rangle_{i^{\prime \prime}} .
$$

Here, $|\psi\rangle_{i^{\prime \prime}}$ and $|\psi\rangle_{i^{\prime \prime}}^{\prime}$ are the states of Bob's qubit $i^{\prime \prime}$. From Eq. (5), it can be seen that if the qubit pair $\left(i, i^{\prime}\right)$ is measured in the Bell states $\left|\phi_{i i^{\prime}}^{+}\right\rangle,\left|\phi_{i i^{\prime}}^{-}\right\rangle,\left|\psi_{i i^{\prime}}^{+}\right\rangle$, and $\left|\psi_{i i^{\prime}}^{-}\right\rangle$, then the states $|\psi\rangle_{i^{\prime \prime}}$ and $|\psi\rangle_{i^{\prime \prime}}^{\prime}$ are, respectively, given by

$$
\begin{aligned}
& |\psi\rangle_{i^{\prime \prime}}=\alpha_{i}|0\rangle_{i^{\prime \prime}}+\beta_{i}|1\rangle_{i^{\prime \prime}},\left|\psi^{\prime}\right\rangle_{i^{\prime \prime}}=\alpha_{i}|1\rangle_{i^{\prime \prime}}-\beta_{i}|0\rangle_{i^{\prime \prime}}, \quad \text { for }\left|\phi_{i i^{\prime}}^{+}\right\rangle \text {, } \\
& |\psi\rangle_{i^{\prime \prime}}=\alpha_{i}|0\rangle_{i^{\prime \prime}}-\beta_{i}|1\rangle_{i^{\prime \prime}},\left|\psi^{\prime}\right\rangle_{i^{\prime \prime}}=\alpha_{i}|1\rangle_{i^{\prime \prime}}+\beta_{i}|0\rangle_{i^{\prime \prime}} \text {, for }\left|\phi_{i i^{\prime}}^{-}\right\rangle \text {, } \\
& |\psi\rangle_{i^{\prime \prime}}=\alpha_{i}|1\rangle_{i^{\prime \prime}}+\beta_{i}|0\rangle_{i^{\prime \prime}},\left|\psi^{\prime}\right\rangle_{i^{\prime \prime}}=-\alpha_{i}|0\rangle_{i^{\prime \prime}}+\beta_{i}|1\rangle_{i^{\prime \prime}} \text {, for }\left|\psi_{i i^{\prime}}^{+}\right\rangle \text {, } \\
& |\psi\rangle_{i^{\prime \prime}}=\alpha_{i}|1\rangle_{i^{\prime \prime}}-\beta_{i}|0\rangle_{i^{\prime \prime}},\left|\psi^{\prime}\right\rangle_{i^{\prime \prime}}=-\alpha_{i}|0\rangle_{i^{\prime \prime}}-\beta_{i}|1\rangle_{i^{\prime \prime}} \text {, for }\left|\psi_{i i^{\prime}}^{-}\right\rangle \text {. }
\end{aligned}
$$

Eq. (9) demonstrates that according to Alice's Bell-state measurement outcome for the pair $\left(i, i^{\prime}\right)$, Bob can restore the original state $\alpha_{i}|0\rangle_{i}+\beta_{i}|1\rangle_{i}$ of the message qubit $i$ from the state $|\psi\rangle_{i^{\prime \prime}}$ or $\left|\psi^{\prime}\right\rangle_{i^{\prime \prime}}$ of his qubit $i^{\prime \prime}$, via a single-qubit Pauli operation $\sigma_{x}, \sigma_{y}$, or $\sigma_{z}$ on the qubit $i^{\prime \prime}$. 
Thirdly, each agent and Alice perform a Hadamard transformation on their respective GHZ qubits. After that, the state (7) becomes [15]

$$
|\psi\rangle\left[\sum_{\left\{x_{l}\right\}}\left|\left\{x_{l}\right\}\right\rangle|0\rangle_{a}+\sum_{\left\{x_{l}\right\}}\left|\left\{y_{l}\right\}\right\rangle|1\rangle_{a}\right]+\left|\psi^{\prime}\right\rangle\left[\sum_{\left\{x_{l}\right\}}\left|\left\{x_{l}\right\}\right\rangle|1\rangle_{a}+\sum_{\left\{y_{l}\right\}}\left|\left\{y_{l}\right\}\right\rangle|0\rangle_{a}\right],
$$

where $\left\{x_{l}\right\}\left(\left\{y_{l}\right\}\right)$ denotes $x_{1} x_{2} \ldots x_{n}\left(y_{1} y_{2} \ldots y_{n}\right)$ and $x_{l}, y_{l} \in\{0,1\}, l=1,2, \ldots, n$ for the $n$ GHZ qubits belonging to the $n$ agents. Furthermore, $\sum_{\left\{x_{l}\right\}}\left|\left\{x_{l}\right\}\right\rangle\left(\sum_{\left\{y_{l}\right\}}\left|\left\{y_{l}\right\}\right\rangle\right)$ sums over all possible basis states $\left|\left\{x_{l}\right\}\right\rangle\left(\left|\left\{y_{l}\right\}\right\rangle\right)$ each containing an even (odd) number of "1"s. For instance, when $n=3, \sum_{\left\{x_{l}\right\}}\left|\left\{x_{l}\right\}\right\rangle=|000\rangle+|110\rangle+|101\rangle+|011\rangle$ while $\sum_{\left\{y_{l}\right\}}\left|\left\{y_{l}\right\}\right\rangle=|001\rangle+|010\rangle+|100\rangle+|111\rangle$.

Lastly, each agent and Alice make a measurement on their respective GHZ qubits, and then send their measurement results to Bob via classical channels. Based on Eq. (10), it is easy to see that Bob can predict whether his $m$ qubits $\left(1^{\prime \prime}, 2^{\prime \prime}, \ldots, m^{\prime \prime}\right)$ are in $|\psi\rangle$ or $\left|\psi^{\prime}\right\rangle$, according to whether the outcomes of the $n$ agents' measurement on their $n$ GHZ qubits contain an even or an odd number of "1"s as well as whether Alice measures her GHZ qubit in the state $|0\rangle$ or $|1\rangle$.

Note that the state $|\psi\rangle\left(\left|\psi^{\prime}\right\rangle\right)$ is a product of individual-qubit states $|\psi\rangle_{1^{\prime \prime}},|\psi\rangle_{2^{\prime \prime}}, \ldots,|\psi\rangle_{m^{\prime \prime}}\left(\left|\psi^{\prime}\right\rangle_{1^{\prime \prime}},\left|\psi^{\prime}\right\rangle_{2^{\prime \prime}}, \ldots,\left|\psi^{\prime}\right\rangle_{m^{\prime \prime}}\right)$ for the qubits $\left(1^{\prime \prime}, 2^{\prime \prime}, \ldots, m^{\prime \prime}\right)$. Hence, Bob can recover the original state $\alpha_{i}|0\rangle_{i}+\beta_{i}|1\rangle_{i}$ of message qubit $i$ from the state $|\psi\rangle_{i^{\prime \prime}}$ or $\left|\psi^{\prime}\right\rangle_{i^{\prime \prime}}$ of his qubit $i^{\prime \prime}$, according to the outcome of Alice's Bell-state measurement on the qubit pair $\left(i, i^{\prime}\right)$ and via a single-qubit Pauli operation $\sigma_{x}, \sigma_{y}$, or $\sigma_{z}$ on the qubit $i^{\prime \prime}$ as described above. Therefore, the quantum information originally carried by the $m$ message qubits $(1,2, \ldots, m)$ can completely be restored by Bob, provided each agent performs a Hadamard transformation followed by a measurement on his/her qubit.

Now let us turn to the next question: What will happen if $k$ agents do not collaborate $(1 \leq k<n)$ ? To answer it, let us return to the state (7). Clearly, this state can be rewritten as

$$
\begin{aligned}
& |\psi\rangle\left[\left(\left|\phi^{+}\right\rangle+\left|\phi^{-}\right\rangle\right)|0\rangle^{\otimes k}+\left(\left|\phi^{+}\right\rangle-\left|\phi^{-}\right\rangle\right)|1\rangle^{\otimes k}\right] \\
& +\left|\psi^{\prime}\right\rangle\left[\left(\left|\phi^{+}\right\rangle+\left|\phi^{-}\right\rangle\right)|0\rangle^{\otimes k}-\left(\left|\phi^{+}\right\rangle-\left|\phi^{-}\right\rangle\right)|1\rangle^{\otimes k}\right],
\end{aligned}
$$

where $|0\rangle^{\otimes k}$ and $|1\rangle^{\otimes k}$ are the two states of the GHZ qubits belonging to the $k$ agents who do not cooperate with Bob, while $\left|\phi^{ \pm}\right\rangle=|0\rangle^{\otimes(n-k)}|0\rangle_{a} \pm|1\rangle^{\otimes(n-k)}|1\rangle_{a}$ are the GHZ states of the remaining $n-k+1$ GHZ qubits belonging to Alice and the other $n-k$ agents who collaborate with Bob.

It is straightforward to show that if the other $n-k$ agents and Alice perform a Hadamard transformation on their respective GHZ qubits, then the states $\left|\phi^{+}\right\rangle$and $\left|\phi^{-}\right\rangle$are transformed into

$$
\begin{aligned}
& \left|\phi^{+}\right\rangle \rightarrow \sum_{\left\{x_{l}^{\prime}\right\}}\left|\left\{x_{l}^{\prime}\right\}\right\rangle|0\rangle_{a}+\sum_{\left\{y_{l}^{\prime}\right\}}\left|\left\{y_{l}^{\prime}\right\}\right\rangle|1\rangle_{a}, \\
& \left|\phi^{-}\right\rangle \rightarrow \sum_{\left\{x_{l}^{\prime}\right\}}\left|\left\{x_{l}^{\prime}\right\}\right\rangle|1\rangle_{a}+\sum_{\left\{y_{l}^{\prime}\right\}}\left|\left\{y_{l}^{\prime}\right\}\right\rangle|0\rangle_{a},
\end{aligned}
$$

where $\left\{x_{l}^{\prime}\right\}\left(\left\{y_{l}^{\prime}\right\}\right)$ denotes $x_{1}^{\prime} x_{2}^{\prime} \ldots x_{n-k}^{\prime}\left(y_{1}^{\prime} y_{2}^{\prime} \ldots y_{n-k}^{\prime}\right)$ with $x_{l}^{\prime}, y_{l}^{\prime} \in\{0,1\}, l=1,2, \ldots, n-k$ for the $n-k$ GHZ qubits belonging to the other $n-k$ agents. And, $\sum_{\left\{x_{l}^{\prime}\right\}}\left|\left\{x_{l}^{\prime}\right\}\right\rangle\left(\sum_{\left\{y_{l}^{\prime}\right\}}\left|\left\{y_{l}^{\prime}\right\}\right\rangle\right)$ sums over all possible basis states $\left|\left\{x_{l}^{\prime}\right\}\right\rangle\left(\left|\left\{y_{l}^{\prime}\right\}\right\rangle\right)$ each containing an even (odd) number of "1"s. Substituting $\left|\phi^{ \pm}\right\rangle$by (12), the state (11) becomes

$$
\begin{aligned}
& {\left[\left(|\psi\rangle+\left|\psi^{\prime}\right\rangle\right)|0\rangle^{\otimes k}+\left(|\psi\rangle-\left|\psi^{\prime}\right\rangle\right)|1\rangle^{\otimes k}\right] \sum_{\left\{x_{l}^{\prime}\right\}}\left|\left\{x_{l}^{\prime}\right\}\right\rangle|0\rangle_{a}} \\
& +\left[\left(|\psi\rangle+\left|\psi^{\prime}\right\rangle\right)|0\rangle^{\otimes k}-\left(|\psi\rangle-\left|\psi^{\prime}\right\rangle\right)|1\rangle^{\otimes k}\right] \sum_{\left\{x_{l}^{\prime}\right\}}\left|\left\{x_{l}^{\prime}\right\}\right\rangle|1\rangle_{a} \\
& +\left[\left(|\psi\rangle+\left|\psi^{\prime}\right\rangle\right)|0\rangle^{\otimes k}-\left(|\psi\rangle-\left|\psi^{\prime}\right\rangle\right)|1\rangle^{\otimes k}\right] \sum_{\left\{y_{l}^{\prime}\right\}}\left|\left\{y_{l}^{\prime}\right\}\right\rangle|0\rangle_{a} \\
& +\left[\left(|\psi\rangle+\left|\psi^{\prime}\right\rangle\right)|0\rangle^{\otimes k}+\left(|\psi\rangle-\left|\psi^{\prime}\right\rangle\right)|1\rangle^{\otimes k}\right] \sum_{\left\{y_{l}^{\prime}\right\}}\left|\left\{y_{l}^{\prime}\right\}\right\rangle|1\rangle_{a},
\end{aligned}
$$


which indicates that if the other $n-k$ agents and Alice perform a measurement on their respective GHZ qubits, the $m$ qubits $\left(1^{\prime \prime}, 2^{\prime \prime}, \ldots, m^{\prime \prime}\right)$ belonging to Bob will be entangled with the $k$ GHZ qubits belonging to the $k$ agents who did not cooperate with Bob.

It is easy to see from Eq. (13) that for every outcome $\left|\left\{x_{l}^{\prime}\right\}\right\rangle|0\rangle_{a},\left|\left\{x_{l}^{\prime}\right\}\right\rangle|1\rangle_{a},\left|\left\{y_{l}^{\prime}\right\}\right\rangle|0\rangle_{a}$, or $\left|\left\{y_{l}^{\prime}\right\}\right\rangle|1\rangle_{a}$ of the other $n-k$ agents' and Alice's measurements on their GHZ qubits, the density operator of Bob's $m$ qubits $\left(1^{\prime \prime}, 2^{\prime \prime}, \ldots, m^{\prime \prime}\right)$ would be given by

$$
\left.\rho=|\psi\rangle\left\langle\psi|+| \psi^{\prime}\right\rangle\left\langle\psi^{\prime}\right|\right)
$$

after tracing over the $k$ GHZ qubits belonging to the $k$ agents who did not cooperate with Bob.

Based on Eqs. (8) and (9), it can be seen that in the case when the qubit pair $\left(t, t^{\prime}\right)$ is measured in the Bell states $\left|\phi_{t t^{\prime}}^{+}\right\rangle,\left|\phi_{t t^{\prime}}^{-}\right\rangle,\left|\psi_{t t^{\prime}}^{+}\right\rangle$, and $\left|\psi_{t t^{\prime}}^{-}\right\rangle$, the states $|\psi\rangle$ and $\left|\psi^{\prime}\right\rangle$ involved in Eq. (14) can be expressed as follows:

$$
\begin{aligned}
& |\psi\rangle=|\widetilde{\psi}\rangle\left(\alpha_{t}|0\rangle_{t^{\prime \prime}}+\beta_{t}|1\rangle_{t^{\prime \prime}}\right),\left|\psi^{\prime}\right\rangle=\left|\widetilde{\psi}^{\prime}\right\rangle\left(\alpha_{t}|1\rangle_{t^{\prime \prime}}-\beta_{t}|0\rangle_{t^{\prime \prime}}\right), \quad \text { for }\left|\phi_{t t^{\prime}}^{+}\right\rangle, \\
& |\psi\rangle=|\widetilde{\psi}\rangle\left(\alpha_{t}|0\rangle_{t^{\prime \prime}}-\beta_{t}|1\rangle_{t^{\prime \prime}}\right),\left|\psi^{\prime}\right\rangle=\left|\widetilde{\psi}^{\prime}\right\rangle\left(\alpha_{t}|1\rangle_{t^{\prime \prime}}+\beta_{t}|0\rangle_{t^{\prime \prime}}\right) \text {, for }\left|\phi_{t t^{\prime}}^{-}\right\rangle \text {, } \\
& |\psi\rangle=|\widetilde{\psi}\rangle\left(\alpha_{t}|1\rangle_{t^{\prime \prime}}+\beta_{t}|0\rangle_{t^{\prime \prime}}\right),\left|\psi^{\prime}\right\rangle=\left|\widetilde{\psi}^{\prime}\right\rangle\left(-\alpha_{t}|0\rangle_{t^{\prime \prime}}+\beta_{t}|1\rangle_{t^{\prime \prime}}\right) \text {, for }\left|\psi_{t t^{\prime}}^{+}\right\rangle \text {, } \\
& |\psi\rangle=|\widetilde{\psi}\rangle\left(\alpha_{t}|1\rangle_{t^{\prime \prime}}-\beta_{t}|0\rangle_{t^{\prime \prime}}\right),\left|\psi^{\prime}\right\rangle=\left|\widetilde{\psi}^{\prime}\right\rangle\left(-\alpha_{t}|0\rangle_{t^{\prime \prime}}-\beta_{t}|1\rangle_{t^{\prime \prime}}\right) \text {, for }\left|\psi_{t t^{\prime}}^{-}\right\rangle \text {, }
\end{aligned}
$$

where the subscript $t^{\prime \prime}$ indicates any one of the $m$ qubits $\left(1^{\prime \prime}, 2^{\prime \prime}, \ldots, m^{\prime \prime}\right)$ belonging to Bob, and the subscripts $t$ and $t^{\prime}$ denote Alice's message qubit and her EPR qubit (corresponding to Bob's qubit $t^{\prime \prime}$ ), respectively. Furthermore, the states $|\widetilde{\psi}\rangle$ and $\left|\widetilde{\psi}^{\prime}\right\rangle$ in Eq. (15) are the states of the remaining $m-1$ qubits belonging to Bob (after excluding the qubit $\left.t^{\prime \prime}\right)$, which are given by $|\widetilde{\psi}\rangle=\prod_{k}|\psi\rangle_{k^{\prime \prime}}$ and $\left|\widetilde{\psi}^{\prime}\right\rangle=\prod_{k}\left|\psi^{\prime}\right\rangle_{k^{\prime \prime}}(k \neq t)$. Here, $|\psi\rangle_{k^{\prime \prime}}$ and $\left|\psi^{\prime}\right\rangle_{k^{\prime \prime}}$ are the states of qubit $k^{\prime \prime}\left(k^{\prime \prime} \neq t^{\prime \prime}\right)$, which depend on the outcome of Alice's Bell-state measurement on the pair $\left(k, k^{\prime}\right)$ and take the form as described by (9). From Eqs. (14) and (15), it can be shown that for each outcome $\left(\left|\phi_{t t^{\prime}}^{+}\right\rangle,\left|\phi_{t t^{\prime}}^{-}\right\rangle,\left|\psi_{t t^{\prime}}^{+}\right\rangle\right.$, or $\left.\left|\psi_{t t^{\prime}}^{-}\right\rangle\right)$of Alice's Bell-state measurement on the pair $\left(t, t^{\prime}\right)$, after tracing over the qubit $t^{\prime \prime}$, the density operator for Bob's remaining $m-1$ qubits is given by

$$
\widetilde{\rho}=\operatorname{tr}_{t^{\prime \prime}}(\rho)=|\widetilde{\psi}\rangle\left\langle\widetilde{\psi}|+| \widetilde{\psi}^{\prime}\right\rangle\left\langle\widetilde{\psi}^{\prime}\right| .
$$

Eq. (16) shows that the density operator, for Bob's remaining $m-1$ "non-traced" qubits, has the same form as (14). Hence, repeating the above single-qubit tracing procedure, we find that the density operator for any qubit $i^{\prime \prime}$ belonging to Bob $\left(i^{\prime \prime}=1^{\prime \prime}, 2^{\prime \prime}, \ldots\right.$, or $\left.m^{\prime \prime}\right)$ can, after tracing over Bob's other $m-1$ qubits, be written as

$$
\rho_{i^{\prime \prime}}=|\psi\rangle_{i^{\prime \prime}}\left\langle\left.\psi\right|_{i^{\prime \prime}}+\mid \psi^{\prime}\right\rangle_{i^{\prime \prime}}\left\langle\left.\psi^{\prime}\right|_{i^{\prime \prime}} .\right.
$$

Based on Eqs. (9) and (17), it is obvious that when Alice measures her qubit pair $\left(i, i^{\prime}\right)$ in either Bell state $\left|\phi_{i i^{\prime}}^{+}\right\rangle$or $\left|\psi_{i i^{\prime}}^{-}\right\rangle$, the density operator $\rho_{i^{\prime \prime}}$ is

$$
\rho_{i^{\prime \prime}}=\frac{1}{2}\left[I+\left(\alpha_{i} \beta_{i}^{*}-\alpha_{i}^{*} \beta_{i}\right)|0\rangle\left\langle 1\left|-\left(\alpha_{i} \beta_{i}^{*}-\alpha_{i}^{*} \beta_{i}\right)\right| 1\right\rangle\langle 0|\right],
$$

where $I=|0\rangle\langle 0|+| 1\rangle\langle 1|$ is an identity. On the other hand, when Alice measures the qubit pair $\left(i, i^{\prime}\right)$ in either Bell state $\left|\phi_{i i^{\prime}}^{-}\right\rangle$or $\left|\psi_{i i^{\prime}}^{+}\right\rangle$, the density operator $\rho_{i^{\prime \prime}}$ is

$$
\rho_{i^{\prime \prime}}=\frac{1}{2}\left[I-\left(\alpha_{i} \beta_{i}^{*}-\alpha_{i}^{*} \beta_{i}\right)|0\rangle\left\langle 1\left|+\left(\alpha_{i} \beta_{i}^{*}-\alpha_{i}^{*} \beta_{i}\right)\right| 1\right\rangle\langle 0|\right] .
$$

In Eqs. (18) and (19) the normalization factor $\frac{1}{2}$ is restored for completeness. The above process demonstrates that the density operator (18) or (19) depends only on the outcome of Alice's Bell-state measurement on the pair $\left(i, i^{\prime}\right)$.

From Eqs. (18) and (19), one sees that the density operator $\rho_{i^{\prime \prime}}$ would be $\frac{1}{2} I$ when $\alpha_{i}$ and $\beta_{i}$ are real. This means that when the message qubit $i$ is initially in the state

$$
\cos \frac{\vartheta_{i}}{2}|0\rangle_{i}+\sin \frac{\vartheta_{i}}{2}|1\rangle_{i},
$$


Bob cannot obtain any amplitude information from his $m$ qubits if there are $k$ agents who do not collaborate. One may worry about a relative phase (dynamic phase) induced due to the free time evolution of the states $|0\rangle$ and $|1\rangle$. However, this phase can be avoided for certain kinds of physical qubits, such as polarized photons or atoms/solid-state devices with degenerate ground levels $|0\rangle$ and $|1\rangle$. It is well known that the polarized photons are important qubit resources for linear optical quantum computation and quantum communication [2,17-23]) and the use of qubits with two degenerate ground levels $|0\rangle$ and $|1\rangle$ is an active way for fault-tolerant quantum computing.

If the initial state of the message qubit $i$ is of the general form

$$
\left|\varphi_{i}\right\rangle=\cos \frac{\vartheta_{i}}{2}|0\rangle_{i}+e^{i \phi_{i}} \sin \frac{\vartheta_{i}}{2}|1\rangle_{i},
$$

the fidelity of the state of qubit $i^{\prime \prime}$ would be

$$
\begin{aligned}
\mathcal{F}_{i^{\prime \prime}} & =\left\langle\varphi_{i}\left|\rho_{i^{\prime \prime}}\right| \varphi_{i}\right\rangle \\
& =\frac{1}{2}\left(1 \pm \sin ^{2} \vartheta_{i} \sin ^{2} \phi_{i}\right)
\end{aligned}
$$

when Alice measures the qubit pair $\left(i, i^{\prime}\right)$ in either of the Bell states $\left|\phi_{i i^{\prime}}^{ \pm}\right\rangle$and $\left|\psi_{i i^{\prime}}^{\mp}\right\rangle$.

To compare the present scheme with the methods in $[5,6,15]$, it is necessary to compute the fidelity averaged over $\vartheta_{i}$ and $\phi_{i}$ (assuming random distributions). A simple calculation finds that the average fidelity would be $\overline{\mathcal{F}}_{i^{\prime \prime}}=2 / 3$ if the qubit pair $\left(i, i^{\prime}\right)$ is measured in either $\left|\phi_{i i^{\prime}}^{+}\right\rangle$or $\left|\psi_{i i^{\prime}}^{-}\right\rangle$. On the other hand, the average fidelity would be $\overline{\mathcal{F}}_{i^{\prime \prime}}=1 / 3$ when the qubit pair $\left(i, i^{\prime}\right)$ is measured in either $\left|\phi_{i i^{\prime}}^{-}\right\rangle$or $\left|\psi_{i i^{\prime}}^{+}\right\rangle$. However, this fidelity can be raised to $2 / 3$ if Bob makes use of the result of Alice's Bell-state measurement. This can be done in the following way. The density matrix of Bob's qubit in Eq. (19) is for two of four results of Alice's Bell-state measurement. If not all agents collaborate Bob does not know if he should apply $\sigma_{x}$ or $\sigma_{z}$ operation. The best he can do is to randomly choose between two possibilities. However, in both cases the result is the same - the density matrix of Eq. (19) is transformed into density matrix of Eq. (18) for which the fidelity is $2 / 3$. Therefore, the average fidelity obtained in the present scheme is equal to that obtained using the methods in $[5,6,15]$. One can verify based on Eqs. (1) and (2) that if the methods in $[5,6,15]$ are used, the average fidelity would be $\overline{\mathcal{F}}_{i^{\prime \prime}}=2 / 3$, independent of the outcomes of the Bell-state measurement for the qubit pair $\left(i, i^{\prime}\right)$. We emphasize that the result obtained above applies to the case of $k=1$. Namely, Bob can retrieve all information only if all agents cooperate.

Several issues may need to be addressed here. Firstly, as a matter of fact, the Hadamard transformations are not necessary for the present proposal. The same results can be obtained when each agent performs a measurement on his/her qubit in the basis $|+\rangle=|0\rangle+|1\rangle$ and $|-\rangle=|0\rangle-|1\rangle$ (instead of a Hadamard transformation and a measurement in the basis $|0\rangle$ and $|1\rangle)$, and then sends his/her measurement result $|+\rangle$ or $|-\rangle$ to the receiver. Secondly, the present proposal actually does not require any specific order among Alice's Bell-state measurement, her single-qubit operation, and each agent's operation. Lastly, the present scheme is secure against eavesdropping and/or cheating as long as Alice sends her classical information to Bob using standard quantum cryptography $[6,15]$. For more discussions, we refer readers to reference [15].

Before conclusion, it should be mentioned that for the present scheme, the resources required for auxiliary qubits, local operations, and classical communication are the same as those needed by the protocol in [15] but are dramatically reduced compared with the methods in [5,6]. This is because similar to the previous proposal in [15] the present scheme only requires that: (i) the sender assigns one qubit to each agent; (ii) each agent performs one single-qubit Hadamard transformation and one single-qubit measurement on his/her qubit; and (iii) each agent sends one-bit classical message to the receiver. For the detail discussion, see Ref. [15].

In summary, we have presented a new scheme for teleporting multiqubit quantum information from a sender to a distant receiver, via the control of many agents in a network. We have shown that for general initial states of each message qubit of arbitrary type, the average fidelity of the output states of the receiver's qubit, when even one agent does not take any action, is equivalent to that of the previous proposals $[5,6,15]$. Therefore, as far as the control of each agent on teleportation for a general input state, the present scheme is as efficient as the proposals $[5,6,15]$. However, as shown above, the present scheme has the distinct feature that for certain kinds of teleported states (20) the receiver cannot gain any amplitude information even if one agent does not collaborate. Note that the states (20) are easily prepared especially for polarized photons (one can modulate the amplitudes by a simple rotation of polarization). Therefore, we believe that the present scheme is of great interest due to its more security, as long as information is encoded through amplitudes of quantum states.

This research was partially supported in part by National Science Foundation ITR program (Grant No. DMR0325551) and AFOSR (Grant No. F49620-01-1-0439), funded under the Department of Defense University Research Initiative on Nanotechnology (DURINT) Program and by the ARDA. 
[1] C. H. Bennett, G. Brassard, C. Crépeau, R. Jozsa, A. Peres, and W. K. Wootters, Phys. Rev. Lett. 70, 1895 (1993).

[2] D. Bouwmeester, J. W. Pan, K. Mattle, M. Eibl, H. Weinfurter, and A. Zeilinger, Nature 390, 575 (1997).

[3] A. Furusawa, J. L. Sørensen, S. L. Braunstein, C. A. Fuchs, H. J. Kimble, and E. S. Polzik, Science 282, 706 (1998).

[4] M. A. Nielsen, E. Knill, R. Laflamme, Nature 396, 52 (1998).

[5] A. Karlsson and M. Bourennane, Phys. Rev. A 58, 4394 (1998).

[6] M. Hillery, V. Buzek, and A. Berthiaume, Phys. Rev. A 59, 1829 (1999).

[7] R. Cleve, D. Gottesman, and H. K. Lo, Phys. Rev. Lett. 83, 648 (1999).

[8] A. Karlsson, M. Koashi, and N. Imoto, Phys. Rev. A 59, 162 (1999).

[9] S. Bandyopadhyay, Phys. Rev. A 62, 012308 (2000).

[10] D. Gottesman, Phys. Rev. A 61, 042311 (2000).

[11] Li-Yi Hsu, Phys. Rev. A 68, 022306 (2003).

[12] A. C. A. Nascimento, J. M. Quade, and H. Imai, Phys. Rev. A 64, 042311 (2001).

[13] B. Aoun, M.Tarifi, e-print, quantum-ph/0401076.

[14] E. Biham, B. Huttner, and T. Mor, Phys. Rev. A 54, 2651 (1996); P. D. Townsend, Nature 385, 47 (1997); S. Bose, V. Vedral, and P. L. Knight, Phys. Rev. A 57, 822 (1998).

[15] C. P. Yang, S. I. Chu, and S. Han, Phys. Rev. A 70, 022329 (2004).

[16] T. Gao, F. L. Yan, Z. X. Wang, quant-ph/0403155; T. Gao, quant-ph/0312004.

[17] E. Knill, R. Laflamme, and G. J. Milburn, Nature 409, 46 (2001).

[18] A. J. F. Hayes, A. Gilchrist, C. R. Myers, T. C. Ralph, quant-ph/0408098.

[19] R. M. Gingrich, P. Kok, H. Lee, F. Vatan, and J. P. Dowling, Phys. Rev. Lett. 91, 217901 (2003).

[20] J. W. Pan, M. Daniell, S. Gasparoni, G. Weihs, and A. Zeilinger, Phys. Rev. Lett. 86, 4435 (2001).

[21] Z. Zhao, Y. A. Chen, A. N. Zhang, T. Yang, H. J. Briegel, and J. W. Pan, Nature 430, 54 (2004).

[22] Y. F. Huang, X. F. Ren, Y. S. Zhang, L. M. Duan, and G. C. Guo, quant-ph/0408007

[23] T. Jennewein, R. Ursin, M. Aspelmeyer, and A. Zeilinger, quant-ph/0409008 